Ann. Zootech., I974, 23 (4), 5I9-536.

\title{
UTILISATION DES PROTÉINES PAR LE VEAU PRÉRUMINANT A L'ENGRAIS
}

\author{
II. - UTILISATION DES PROTÉINES DE POISSON \\ ET INFLUENCE DE L'ADDITION D'UN COMPLEXANT DU FER
}

\author{
J.-L. PARUELLE, R. TOULLEC, \\ P. PATUREAU-MIRAND* et C.-M. MATHIEU \\ avec la collaboration technique de Marguerite BEAurils, \\ J. LAREYnie, Yvette Lento et Y. Manis \\ Station de Recherches zootechniques, \\ Centre de Recherches de Rennes, I. N.R. A., \\ 65, Rue de Saint-Brieuc \\ 35042 Rennes Cedex \\ * Laboratoire d'Études du Métabolisme azoté, \\ Centre de Recherches de Clevmont-Ferrand, I. N. R. A., \\ Theix, 63110 Beaumont
}

\section{RÉSUMÉ}

Nous avons étudié l'utilisation des protéines de poisson dans les aliments d'allaitement destinés au Veau préruminant à l'engrais. Ces protéines étaient apportées sous la forme d'un concentré obtenu par hydrolyse enzymatique ménagée du poisson blanc et contenant environ 90 p. 100 de matières azotées et 80 p.p.m. de fer.

Dans une première expérience, 3 veaux, maintenus en cages à bilans, ont reçu un aliment d'allaitement dont 73 p. Ioo des protéines provenaient du poisson, le reste étant apporté par du lactosérum et un supplément de méthionine. La fréquence des jours de diarrhée a été très faible ( 2 p. IOo), celle des jours d'état relâché a été importante (74 p. I0o). La digestibilité apparente des matières azotées de l'aliment a augmenté au cours du premier mois d'âge, de 82 p. Ioo entre I 5 et 2 I jours, à 89 p. Ioo entre 24 et 34 jours. Elle a ensuite été élevée et constante ( 92 p. IOo) entre 37 et ro5 jours. Cette évolution traduirait une adaptation insuffisante du système digestif du très jeune veau à la modification du transit gastro-intestinal due à l'absence de coagulation des protéines dans la caillette. Le coefficient de rétention apparent de l'azote a été élevé, montrant que l'équilibre en acides aminés indispensables disponibles des protéines alimentaires était satisfaisant.

Dans une deuxième expérience, 48 veaux ont été élevés en cases individuelles depuis l'âge d'environ une semaine, jusqu'au poids vif d'abattage d'environ I $50 \mathrm{~kg}$. Après une semaine d'adaptation, ils ont été répartis en 5 lots et ont reçu l'un des 5 aliments d'allaitement étudiés; dans le premier aliment ( 16 veaux), les protéines provenaient exclusivement du lait. Dans les deux suivants ( 2 lots de 8 veaux), elles provenaient pour 68 p. roo du poisson, le reste étant apporté par du lactosérum et un supplément de méthionine. L'un d'eux était additionné d'un composé de l'EDTA, de façon à complexer le fer excédentaire provenant du poisson. La composition des deux autres aliments ( 2 lots de 8 veaux) était intermédiaire entre le premier aliment et chacun des deux suivants. Tous les aliments ont été bien consommés et l'état sanitaire des animaux a été satisfaisant. 
L'aliment dont les protéines provenaient du lait et ceux ayant une composition intermédiaire ont permis des gains de poids vif voisins (environ I Ioo g/j) et significativement supérieurs à ceux obtenus avec les aliments dont $68 \mathrm{p}$. Ioo des protéines provenaient du poisson ( $\mathrm{I}$ oog g/j). La quantité de matière sèche ingérée par $\mathrm{kg}$ de gain de poids vif a significativement augmenté avec le taux de substitution des protéines du lait (respectivement $\mathbf{I}, 48-\mathbf{I}, 55$ et $\mathrm{I}, 67 \mathrm{~kg}$ ). Le rendement à l'abattage et l'état d'engraissement des carcasses ont été satisfaisants dans tous les cas. La coloration de la viande a été légèrement trop foncée chez les veaux qui recevaient l'aliment riche en protéines de poisson non additionné de complexant du fer, et satisfaisant dans tous les autres cas. D'après l'évolution de l'hématocrite des veaux, le complexant du fer semble avoir limité l'utilisation du fer alimentaire.

Les protéines issues du poisson blanc et ayant subi une hydrolyse enzymatique ménagée semblent intéressantes à incorporer dans les aliments d'allaitement. Associées au lactosérum, elles pourraient apporter environ la moitié des protéines alimentaires sans occasionner de baisse dans les performances zootechniques des veaux.

\section{INTRODUC'TION}

Dans les aliments d'allaitement actuellement utilisés pour l'élevage et l'engraissement du Veau, une partie du lait écrémé est parfois remplacée par d'autres sources de protéines. Pour être bien utilisées, celles-ci doivent présenter un équilibre satisfaisant en acides aminés indispensables et ne pas contenir de constituants peu digestibles (glucides complexes de certains produits végétaux) ou indésirables (facteurs antinutritionnels...). Elles doivent en outre être solubles ou présenter un bon maintien en suspension (ToulLEC et al., I973).

Le poisson constitue une source intéressante de protéines d'origine animale, disponibles sous forme concentrée $(70$ à $90 \mathrm{p}$. IOO). Ses protéines sont riches en lysine, mais légèrement carencées en acides aminés soufrés (Pion et Fauconneau, I966). Leur incorporation dans l'alimentation lactée du Veau a surtout été envisagée dans le cas des veaux sevrés précocement (HARshbarger et Geiwicks, ig65; Huber et Slade, r967; Genskow, Harshbarger et Wendrandt, ig68 et ig69; Gorrili, Nicholson et Power, I972;.Khammouma et Arbogast, I970; MaKDANi, Huber et Michei, I97I; Michei, et al., I972; RAVEN, I972; WeNdiANdT, HARShbarger et Genskow, I968).

Nous avons donc étudié l'utilisation des protéines de poisson par le Veau préruminant à l'engrais. Dans une première expérience, l'utilisation digestive et métabolique d'un lait de remplacement, dont les protéines provenaient en majeure partie du poisson, a été mesurée chez des veaux maintenus en cages à bilans entre les âges d'environ 8 et Ioo j. Dans une deuxième expérience, la croissance et les résultats d'abattage ont été étudiés sur des veaux élevés en cases individuelles jusqu'au poids vif de I50 kg et recevant 3 laits de remplacement différant par l'origine de leurs protéines : dans l'un, elles provenaient exclusivement du lait, dans le deuxième, elles provenaient en grande partie du poisson et dans le troisième, leur composition était intermédiaire entre celle des deux précédents. Chez le Veau préruminant à l'engrais, un certain état d'anémie est nécessaire pour obtenir une viande peu colorée. Du fait de la teneur un peu élevée en fer du concentré de protéines de poisson utilisé, l'influence de la supplémentation des deux derniers laits de remplacement par un complexant du fer a également été envisagée. 


\section{MATÉRIEI, ET MÉTHODES}

Nous avons étudié l'utilisation d'un concentré protéique, obtenu à partir de poissons blancs, par hydrolyse enzymatique ménagée, suivie d'abord de deux centrifugations pour éliminer successivement les protéines demeurées insolubles et une partie des lipides, puis d'un séchage par le procédé Spray. Le produit ainsi préparé (CPSP 9o) est soluble et contient environ 89 p. Ioo de matières azotées, 6 p. Ioo de lipides, 6 p. Ioo de matières minérales et 80 p.p.m. de fer.

La composition en acides aminés du concentré protéique a été déterminée par chromatographie sur colonne, selon les techniques de PION et Fauconveau (I966). Le tryptophane a été dosé à l'aide d'une technique dérivée de celle de Slump et Schreuder (I969). Les compositions correspondantes des aliments ont été calculées à partir de la composition moyenne de leurs constituants azotés déterminée selon les mêmes techniques. Les matières azotées du concentré protéique sont relativement bien équilibrées en acides aminés indispensables, quoique carencées en acides aminés soufrés, en tryptophane et/ou en acides aminés aromatiques et histidine (tabl. I). La somme des teneurs corrigées en acides aminés indispensables et semi-indispensables est peu élevée ( 37 au lieu de 52 p. Ioo des acides aminés totaux pour les protéines du lait).

TABLEAU I

Teneur en acides aminés du concentré protéique de Poisson ( $\mathrm{g}$ p. I6 g N) (PION : communication personnelle).

\begin{tabular}{|c|c|c|c|}
\hline Ac. aspartique ... & 8,65 & Méthionine ....... & 2,5 \\
\hline Thréonine .......... & 3,8 & Isoleucine $\ldots . . .$. & 3,5 \\
\hline Sérine $\ldots \ldots \ldots \ldots$ & 4,45 & Leucine.......... & 6,05 \\
\hline Ac. glutamique $\ldots .$. & 12,85 & Tyrosine & 2,45 \\
\hline Proline $\ldots \ldots \ldots$ & 4,55 & Phénylalanine. & 2,95 \\
\hline Glycine.......... & 8,0 & Lysine....... & 7,6 \\
\hline Alanine...$\ldots \ldots$ & 6,2 & Histidine & 1,95 \\
\hline Valine............ & 4,25 & Arginine & 6,3 \\
\hline Cystine $\ldots \ldots \ldots \ldots$ & 0,75 & Tryptophane ...... & 0,55 \\
\hline \multicolumn{3}{|c|}{$\begin{array}{l}\text { Somme des teneurs corrigées }\left({ }^{1}\right) \text { en acides aminés indispen- } \\
\text { sables et semi-indispensables }\end{array}$} & $37, \mathbf{k}$ \\
\hline
\end{tabular}

(1) Teneur en leucine limitée à 1,5 fois celle en isoleucine; somme des teneurs en tyrosine et phénylalanine limitée à 12 p. 100 de la somme des acides aminés indispensables et semi-indispensables; teneur en arginine limitée à 7,3 p. 100 de la somme des teneurs en acides aminés indispensables et semi-indispensables.

\section{Expérience de bilans}

Nous avons utilisé un aliment d'allaitement (aliment Poisson) dont environ 73 p. Ioo des matières azotées provenaient du poisson, le reste étant apporté par du lactosérum et un supplément de méthionine (tabl. 2). Le lactosérum permettait l'introduction de lactose et servait de support pour les matières grasses. L'aliment ne contenait pas de lait écrémé et ne coagulait pas sous l'action de la présure et de l'acidité gastrique.

L'essai a été effectué sur 3 veaux mâles de race frisonne, achetés à l'âge d'environ une semaine et placés dans des cages à bilans dès leur arrivéc. Les mesures ont commencé une semaine plus tard, de manière à laisser les animaux s'adapter à leurs nouvelles conditions d'existence. Elles ont alors été effectuées pendant une période de 4 jours, suivie de 6 périodes de r r jours, séparées par des interpériodes de 3 jours. Les modalités de préparation et de distribution du lait de remplacement, ainsi que les méthodes de prélèvement, de mesure, de dosage et de calcul, ont été décrites précédemment (PARuelle et al., I972). 
TABLEAU 2

Composition des aliments

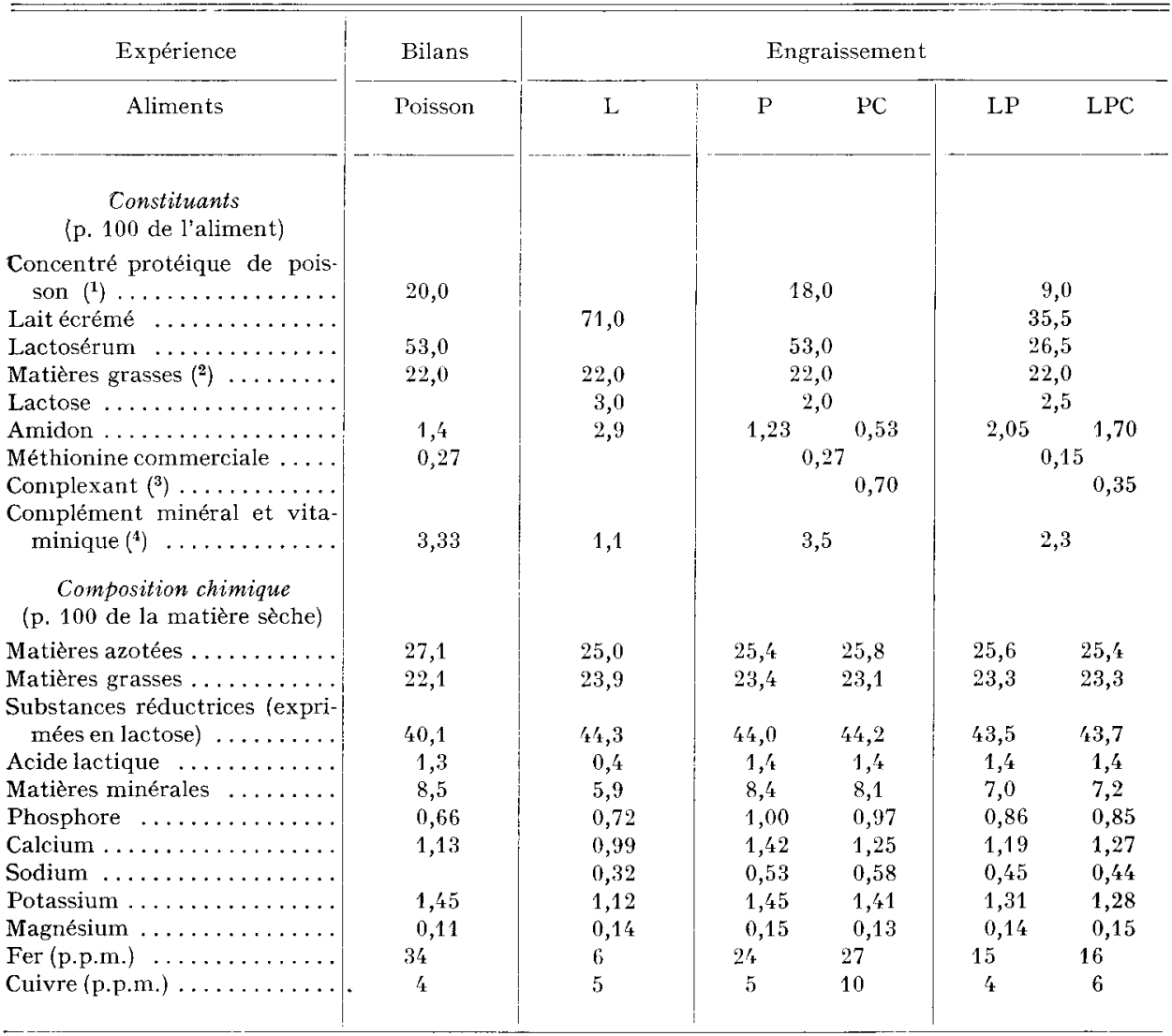

(1) CPSP 90, de la Coopérative de Traitement des produits de la Pêche. B. P. 361/2, 62200 Boulognesur-Mer.

(2) Les matières grasses étaient constituées d'un mélange de suif (aliment Poisson : 73 p. 100 , autres aliments : 78 p. 100), d'huile de coprah (aliment Poisson : 25 p. 100, autres aliments : 20 p. 100) et de saccharoglycérides (2 p. 100). Elles ont été incorporées à raison de 35 p. 100 de la matière sèche exclusivement dans du lactosérum liquide (aliment Poisson, $\mathrm{P}$ et $\mathrm{PC}$ ) ou du lait écrémé liquide (aliment $\mathrm{L}$ ) ou pour moitié dans chacun de ces 2 produits (aliments LP et LPC). Les mélanges obtenus ont été homogénéisés et séchés par le procédé Spray.

$\left.{ }^{3}\right)$ Nourycal 25, de la Société Akzo Chimie France, 9, rue Mathurin Régnier, 75015 Paris.

(4) La composition des compléments vitaminiques et antibiotiques était la même que celle de ceux des aliments Levures et Soja (Paruelle et al., 1972).

\section{Expérience d'engraissement}

Nous avons comparé 5 aliments d'allaitement ne différant que par l'origine de leurs protéines et par leur supplémentation éventuelle en un complexant du fer (tabl. 2).

Dans l'un de ces aliments (aliment L), les matières azotées étaient apportées exclusivement par du lait écrémé. Dans deux autres aliments (aliment $\mathrm{P}$ et aliment $\mathrm{PC}$ ), elles provenaient pour 
68 p. roo du concentré de protéines de poisson déjà utilisé dans l'expérience de bilans et pour 32 p. roo du lactosérum et d'un supplément de méthionine. La composition de ces aliments était donc très voisine de celle de l'aliment Poisson. L'un d'eux (aliment PC) était additionné d'un dérivé calcique et disodique de l'acide éthylène diamine tétracétique (EDTA), destiné à complexer l'apport de fer alimentaire provenant du concentré de protéines de poisson $(0,7$ p. Ioo de complexant dans l'aliment pour 20 p.p.m. de fer). La composition des 2 derniers aliments (aliments LP et LPC) était intermédiaire entre celle des aliments $L$ d'une part et $P$ ou PC d'autre part.

Dans tous ces aliments, les teneurs en acides aminés indispensables et semi-indispensables étaient d'autant plus faibles que le taux de lait écrémé séché était bas (tabl. 3). Les matières azotées des aliments $\mathrm{P}$ et $\mathrm{PC}$, supplémentées en méthionine, paraissaient bien équilibrées en acides aminés. Seule aurait subsisté une légère carence en tryptophane et/ou en acides aminés aromatiques et en histidine.

TABLEAU 3

Composition en acides aminés des aliments $\left({ }^{\mathbf{1}}\right)$

(g p. I6 g N)

\begin{tabular}{|c|c|c|c|c|}
\hline Aliments & Poisson & P et $\mathrm{PC}$ & LP et $\mathrm{LPC}$ & $\mathrm{L}$ \\
\hline Ac. aspartique $\ldots \ldots \ldots$ & 8,65 & 8,65 & 8,25 & 7,9 \\
\hline Thréonine $\ldots \ldots \ldots \ldots$ & 4,4 & 4,4 & 4,5 & 4,6 \\
\hline Sérine $\ldots \ldots \ldots \ldots \ldots$ & 4,45 & 4,45 & 5,15 & 5,8 \\
\hline Ac. glutamique $\ldots \ldots \ldots$ & 13,5 & 13,55 & 18,25 & 22,6 \\
\hline Proline ............ & 4,75 & 4,8 & 7,45 & 9,9 \\
\hline Glycine $\ldots \ldots \ldots \ldots \ldots$ & 6,2 & 6,05 & 3,95 & 2,0 \\
\hline Alanine . . . . . . . . . & 5,55 & 5,5 & 4,45 & 3,5 \\
\hline Valine $\ldots \ldots \ldots \ldots \ldots$ & 4,55 & 4,6 & 5,9 & 7,15 \\
\hline Cystine...$\ldots \ldots \ldots$. & 0,95 & 0,95 & 0,95 & 0,9 \\
\hline Méthionine $\ldots \ldots \ldots \ldots$ & 3,35 & 3,4 & 3,05 & 2,6 \\
\hline Isoleucine $\ldots \ldots \ldots \ldots \ldots$ & 4,05 & 4,1 & 4,95 & 5,75 \\
\hline Leucine $\ldots . . . \ldots \ldots$ & 6,75 & 6,8 & 8,45 & 10,0 \\
\hline Tyrosine ............ & 2,45 & 2,5 & 3,85 & 5,1 \\
\hline Phénylalanine ........ & 3,05 & 3,05 & 4,25 & 5,35 \\
\hline Lysine $\ldots . . . . .$. & 7,65 & 7,65 & 8,1 & 8,5 \\
\hline Histidine $\ldots \ldots \ldots \ldots$ & 1,85 & 1,85 & 2,4 & 2,9 \\
\hline Arginine $\ldots \ldots \ldots \ldots$ & 5,05 & 4,95 & 4,25 & 3,55 \\
\hline Tryptophane ......... & 0,8 & 0,8 & 1,0 & 1,15 \\
\hline $\begin{array}{l}\text { Somme des teneurs corri- } \\
\text { gées en acides aminés } \\
\text { indispensables et semi- } \\
\text { indispensables }\end{array}$ & 41,75 & 42,0 & 47,43 & 51,95 \\
\hline
\end{tabular}

(1) Composition calculée à partir des valeurs du tableau 2 et de la composition en acides aminés des autres composés protéiques de l'aliment (Pron et Fauconneau, 1966; Pion, 1971).

Quarante-huit veaux mâles de race Frisonne ont été achetés à l'âge d'environ une semaine. Dès leur arrivée, ils ont été munis d'une muselière et placés en cases individuelles sur litière de paille dans une étable non chauffée. Après une période d'observation de 6 jours, les animaux ont été répartis en I lot de 16 veaux et 4 lots de 8 veaux aussi semblables que possible compte tenu de leur poids vif, de leur gain de poids vif, de la valeur de leur hématocrite et de leur état sanitaire. Pendant la période d'adaptation, les animaux ont tous reçu un aliment d'allaitement du commerce auquel a été substitué l'un des 5 aliments expérimentaux entre le neuvième et le douzième jour. Ces aliments ont été distribués à I 6 animaux (aliment L) ou à 8 animaux (aliments 
$\mathbf{P}, \mathbf{P C}, \mathrm{LP}$ et LPC). Au cours des deux premières semaines après l'arrivée, les veaux ont reçu des quantités modérées de lait de remplacement, puis ils ont été alimentés en fonction de leur poids vif et de leur appétit (tabl. 4).

\section{TABLEAU 4}

Expérience d'engraissement : plan de rationnement

\begin{tabular}{|c|c|c|c|}
\hline \multirow{2}{*}{$\begin{array}{l}\text { Poids vif } \\
\text { à la dernière pesée } \\
\text { hebdomadaire }(\mathrm{kg})\end{array}$} & \multicolumn{2}{|c|}{$\begin{array}{l}\text { Quantité d'aliment proposée } \\
\text { (g/jour) }\end{array}$} & \multirow{2}{*}{$\begin{array}{c}\text { Taux } \\
\text { de reconstitution } \\
\text { (g d'aliment/kg de lait } \\
\text { de remplacement) }\end{array}$} \\
\hline & maximum & minimum & \\
\hline 40 & \multicolumn{2}{|c|}{910} & 130 \\
\hline 50 & \multicolumn{2}{|c|}{1040} & 130 \\
\hline 60 & 1300 & 1170 & 130 \\
\hline 70 & 1560 & 1300 & 130 \\
\hline 80 & 1680 & 1400 & 140 \\
\hline 90 & 1950 & 1650 & 150 \\
\hline 100 & 2240 & 1760 & 160 \\
\hline 110 & 2380 & 1870 & 170 \\
\hline 120 & 2700 & 2160 & 180 \\
\hline 130 & 2850 & 2280 & 190 \\
\hline 140 & 3040 & 2280 & 190 \\
\hline
\end{tabular}

Les animaux ont été pesés dès leur arrivée, tous les 7 jours à heure fixe et avant l'abattage qui a lieu au poids vif d'environ I $_{5} 0 \mathrm{~kg}$, soit de 9 I à I I9 jours après leur arrivée. Des prélèvements de sang ont été effectués dans la veine jugulaire sur tous les veaux au cours de la première semaine, à la fin du premier, du deuxième, du troisième mois d'engraissement et avant l'abattage, pour la détermination de la valeur de l'hématocrite.

Après chaque repas, les refus alimentaires individuels ont été pesés. Pour chaque veau, le gain de poids vif et l'efficacité alimentaire ont été mesurés à partir du huitième jour d'engraissement. Lors de l'abattage, la carcasse chaude, les différentes parties de l'appareil digestif plein et vide, les dépôts adipeux périrénaux, péritonéaux, le foie, les reins et le pancréas ont été pesés. Des prélèvements de muscle au niveau de l'onglet (piliers latéraux du diaphragme) ont été effectués en vue de déterminer leur teneur en fer héminique d'après la méthode de HoRNSEY (I956). Un morceau du carré couvert a été prélevé au même endroit sur la carcasse d'un veau du lot L et celle d'un veau du lot P. Six jours plus tard, après avoir été débités et frits au beurre, chacun d'eux a été soumis sans identification à $I 7$ personnes afin de rechercher d'éventuelles modifications du goût occasionnées par le remplacement des protéines du lait par celles du poisson.

\section{RÉSULTATS}

\section{Expérience de bilans (tabl. 5)}

Nous n'avons pas tenu compte, dans 1'expression des quantités consommées des gains de poids vif et de l'efficacité alimentaire, des résultats obtenus avec l'un des veaux qui avait eu une croissance anormalement faible $(805 \mathrm{~g} / \mathrm{j})$ par suite de troubles d'arthrite. Les deux autres animaux ont très bien consommé l'aliment Poisson au cours des 2 premiers mois de l'essai (environ 3 p. Ioo de refus) ; au cours du troisième mois, ils ont refusé 20 p. Ioo des quantités proposées, probablement 
par suite d'un niveau de rationnement excessif. Les gains de poids vif obtenus ont été satisfaisants $(983 \mathrm{~g} / \mathrm{j})$ mais l'efficacité alimentaire a été médiocre $(\mathrm{I}, 70 \mathrm{~kg}$ de matière sèche ingérée/kg de gain de poids vif).

\section{TABLEAU 5}

\section{Expérience de bilans : résultats}

\begin{tabular}{|c|c|c|c|}
\hline & & 1 & \\
\hline $\begin{array}{c}\text { Quantité de matière sèche ingérée } \\
(\mathrm{kg})\left({ }^{1}\right) \ldots \ldots \ldots \ldots \ldots \ldots \ldots \ldots\end{array}$ & 145,5 & $\begin{array}{l}\text { Coefficient d'utilisation digestive } \\
\text { apparent (p. 100) }\left({ }^{3}\right)\end{array}$ & \\
\hline Gain de poids vif $(\mathrm{g} / \mathrm{j})(\mathbf{l}) \ldots \ldots \ldots$ & 983 & - matière sèche............ & $\pm 1,4$ \\
\hline Quantités ingérées par $\mathrm{kg}$ de gain & & - matiere organique.......... & $95,2 \pm 1,3$ \\
\hline de poids vif $(\mathbf{1}):$ & & - matières azotées ........... & $91,9 \pm 1,4$ \\
\hline - matière sèche $(\mathrm{kg})$. & & - matières grasses .. & $92,5 \pm 3,0$ \\
\hline - matière organique digestible $(\mathrm{kg})$ & 1,47 & - matières minérales ........ & $87,5 \pm 2,6$ \\
\hline - énergie digestible (Mcal) ${ }^{(2)} \ldots .$. & 7,86 & Coefficient de rétention apparent de & \\
\hline Énergie digestible ingérée sous forme & & l'azote $\left(\right.$ p. 100) $\left(^{(4)} \ldots \ldots \ldots \ldots\right.$ & $65,2 \pm 5,9$ \\
\hline $\begin{array}{l}\text { de matières azotées (p. } 100 \quad \text { de } \\
\text { l'énergie digestible ingérée) }(\mathbf{1}) \ldots\end{array}$ & $24, t_{k}$ & $\begin{array}{c}\text { Coefficient d'utilisation pratique } \\
\text { de l'azote }(p .100)\left({ }^{4}\right) \ldots \ldots \ldots \ldots\end{array}$ & $58,8 \pm 6,6$ \\
\hline & & $\begin{array}{l}\text { Quantité d'azote apparemment rete- } \\
\text { nue par } \mathrm{kg} \text { de gain de poids vif } \\
(\mathrm{g})\left({ }^{1}\right) \ldots \ldots \ldots \ldots \ldots \ldots \ldots \ldots \ldots\end{array}$ & 44,2 \\
\hline
\end{tabular}

(1) Au cours des 87 jours d'expérience.

( $\left.{ }^{2}\right)$ Calculée en admettant les valeurs calorifiques suivantes :

9,$10 ; 5,65 ; 3,85 ; 4,18$ pour les matieres grasses, les matières azotćes, le lactose et le reste de la fraction glucidique respectivement (d'après BLAxTER, 1962).

${ }^{3}$ ) Moyenne et écart-type des 5 dernières périodes de mesure.

(4) Moyenne et écart-type des 7 périodes de mesure.

L'état diarrhéique a été caractérisé d'après la classification basée sur la teneur en matière sèche des fèces proposée par BLAXTER et WOOD (I953). La fréquence des jours de diarrhée a été très faible pendant toute la durée de l'expérience (2,I p. I00), mais celle des jours d'état relâché a été importante (74,I p. I00). L'état sanitaire a cependant toujours été satisfaisant, sauf pour l'animal atteint d'arthrite,

Le coefficient d'utilisation digestive (CUD) apparent des matières azotées a d'abord augmenté avec l'âge des animaux, passant ainsi de 82 p. Ioo au cours de la première période de mesure, à $89 \mathrm{p}$. Ioo au courrs de la seconde et à $92 \mathrm{p}$. Ioo au cours de la troisième, à partir de laquelle il est resté sensiblement constant (fig. I). En utilisant la valeur ( 94 p. Ioo) obtenue pour le CUD apparent des matières azotées du lactosérum enrichi par ultrafiltration (TOULLEC et al., résultats non publiés). le CUD apparent des matières azotées du concentré protéique de poisson peut être estimé à $9 \mathrm{I}$ p. Ioo à partir de l'âge d'un mois. Le coefficient de rétention apparent de l'azote a été élevé $(65,2 \mathrm{p}$. Ioo) et a eu légèrement tendance à diminuer avec l'âge des veaux.

Les CUD apparents de la matière sèche, de la matière organique, des matières grasses et des matières minérales ont varié avec l'âge des animaux, de la même manière que celui des matières azotées (fig. I). 

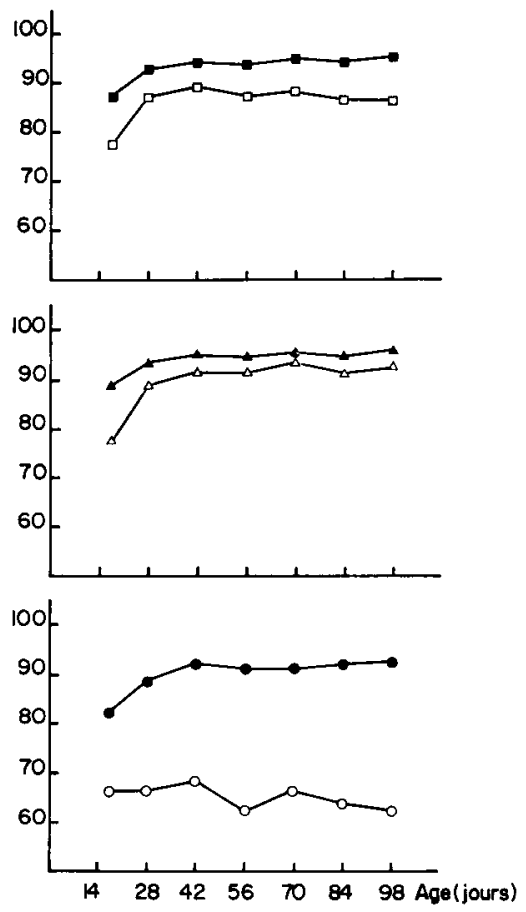

FIG. I. - Evolution des coefficients d'utilisation digestive apparents et du coefficient de rétention apparent de l'azote avec l'âge

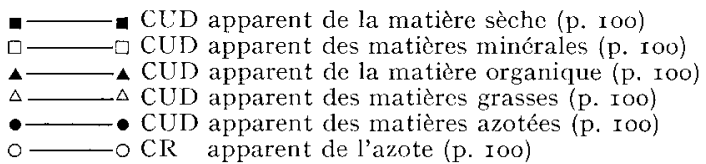

\section{État sanitaire.}

\section{Expérience d'engraissement}

Deux animaux sont décédés à la suite de météorisations : l'un, du lot PC et l'autre, du lot LP, respectivement après 66 et 36 jours de présence. Par ailleurs, deux animaux ont etı des performances très faibles. L'un, du lot $L$, a été éliminé au bout de 84 jours d'essai alors que son gain de poids vif journalier moyen était de 179 g. L'autre, du lot $\mathrm{P}$, dont la valeur de l'hématocrite était de 22 au début de l'essai, a été abattu à $\mathrm{I} 32 \mathrm{~kg}$ de poids vif, à 1 'issue du $\mathrm{II} 8^{\mathrm{e}}$ jour de présence et n'a pas été retenu dans l'expression des résultats moyens.

\section{Quantités ingérées.}

Les aliments ont été bien consommés puisque, sur l'ensemble de l'essai, les quantités refusées par rapport aux quantités proposées n'ont été que de 2,4 p. Ioo pour les lots $\mathrm{P}$ et $\mathrm{PC}$ réunis, de $0,8 \mathrm{p}$. roo pour les lots LP et LPC réunis et de 3,7 p. Ioo pour le lot L. Dans ce dernier cas, la plus forte proportion des refus a été due à la présence d'un animal ayant refusé I3 p. Ioo des quantités proposées. Par 
ailleurs, la supplémentation en complexant du fer n'a pas eu d'influence importante sur la fréquence des refus alimentaires. Ceux-ci ont été de 3,4 p. Ioo pour le lot PC contre I,5 p. Ioo pour le lot $\mathrm{P}$, et de $0,7 \mathrm{p}$. Ioo pour le lot LPC contre $0,9 \mathrm{p}$. Ioo pour le lot L.P.

Gain de poids vif et efficacité alimentaire.

Au cours des 8 premières semaines d'essai, les animaux des lots P et PC ont réalisé des gains de poids vif voisins (tabl. 6), avec des efficacités alimentaires exprimées en quantité de matière sèche ingérée par $\mathrm{kg}$ de gain de poids vif, semblables. Au cours des 4 semaines suivantes, les animaux du lot $\mathrm{P}$ ont effectué une croissance plus rapide que ceux du lot $\mathrm{PC}(p<0,05)$ avec une meilleure efficacité alimentaire (non significatif), probablement par suite d'un état d'anémie moins prononcé. Il en a été de même entre les animaux des lots LP et LPC sans qu'aucune différence significative n'ait été observée.

Toutefois, sur l'ensemble de l'essai, les performances des animaux des lots $\mathbf{P}$ et PC, de même que celles des veaux des lots LP et LPC, dont l'alimentation ne différait que par une supplémentation ou non en complexant du fer, ont été voisines. En conséquence, l'étude de l'influence de l'origine des protéines alimentaires sur la croissance et l'efficacité alimentaire, a pu être entreprise en regroupant les animaux des lots $\mathrm{P}$ et PC et ceux des lots LP et LPC.

Au cours des 4 premières semaines d'essai, les veaux des lots témoin et $\mathrm{LP}+\mathrm{LPC}$ ont eu des gains de poids vif semblables, avec des efficacités alimentaires très voisines. Les 4 semaines suivantes, des résultats moins satisfaisants (NS) ont été obtenus par les veaux du lot $\mathrm{L}_{1} \mathrm{P}+\mathrm{LPC}$, alors que de la neuvième à la douzième semaine, les performances des animaux des 2 lots ont été très voisines. Au cours de chacune de ces 3 périodes, la croissance des animaux du lot $\mathrm{P}+\mathrm{PC}$ a toujours été inférieure à celle des veaux du lot témoin $(p<0$,or de la cinquième à la huitième semaine), ainsi qu'à celle des animaux du lot $\mathrm{LP}+\mathrm{LPC}(p<0,05$ de la cinquième à la huitième semaine et $p<0$, or de la neuvième à la douzième semaine). De plus, l'efficacité alimentaire a été moins satisfaisante chez ces animaux que chez ceux du lot témoin $(p<0$, or les 4 premières semaines et de la cinquième à la huitième semaine) et du lot LP + LPC $(p<0,05$ de la cinquième à la huitième semaine).

Au cours des 12 premières semaines d'essai, comme sur 1'ensemble de l'expérience, les animaux des lots L et LP + LPC ont effectué des gains de poids vif très voisins. Si l'efficacité alimentaire a été significativement moins bonne chez les animaux du lot LP + LPC, lorsqu'elle était exprimée en matière sèche $(p<0,05)$, la différence disparaissait lorsqu'elle était exprimée en matière organique digestible. Par ailleurs, la croissance et l'efficacité alimentaire ont été moins satisfaisantes $(p<0,0 r)$ pour les animaux du lot $\mathrm{P}+\mathrm{PC}$ que pour ceux des lots $\mathrm{L}$ et $\mathrm{LP}+\mathrm{LPC}$. Les animaux de ces 2 derniers lots sont parvenus au poids vif d'environ I50 $\mathrm{kg}$ à l'issue de durées d'essai comparables (95 et 93 j). En revanche, les animaux du lot $\mathrm{P}+\mathrm{PC}$ ne sont parvenus à un poids vif de $\mathrm{I} 47 \mathrm{~kg}$ qu'à l'issue d'une durée d'essai supérieure (IOO j).

Évolution de la valeur de l'hématocrite (tab1. 7).

Les valeurs de l'hématocrite des veaux recevant l'aliment $L$, très pauvre en fer (6 p.p.m.) et les aliments PC et LPC, supplémentés en complexant du fer, ont 


\begin{tabular}{|c|c|c|c|c|c|c|c|}
\hline \multirow{8}{*}{ 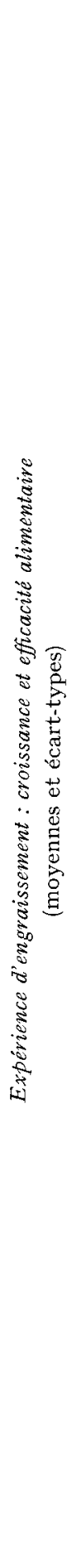 } & $\begin{array}{l}0 \\
\vdots \\
+ \\
+ \\
2 \\
2\end{array}$ & 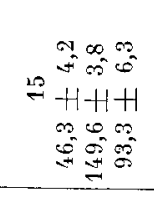 & 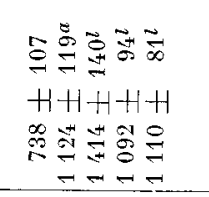 & $\begin{array}{l}0 \\
\infty \\
\infty \\
+1 \\
0 \\
0 \\
0 \\
0\end{array}$ & & 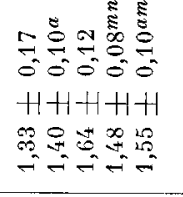 & $\begin{array}{l}8 \\
8 \\
+1 \\
\text { के } \\
-\end{array}$ \\
\hline & $\begin{array}{l}u \\
\dot{Q} \\
+ \\
D_{4}\end{array}$ & 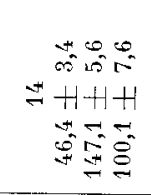 & 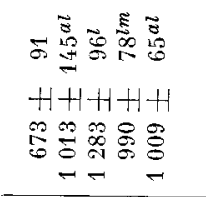 & $\begin{array}{l}+1 \\
\infty \\
+\infty \\
+1 \\
\infty \\
0 \\
0 \\
0\end{array}$ & & 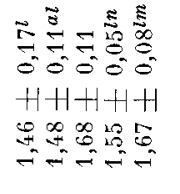 & $\begin{array}{l}8 \\
8 \\
H \\
H \\
0 \\
0 \\
\Rightarrow\end{array}$ \\
\hline & $\underset{\Xi}{J}$ & 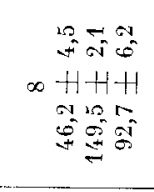 & 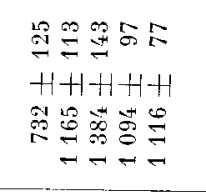 & $\begin{array}{l}\infty \\
\infty \\
+1 \\
0 \\
0 \\
0 \\
0\end{array}$ & & 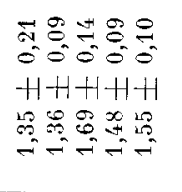 & 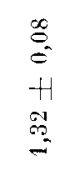 \\
\hline & $\stackrel{a}{a}$ & 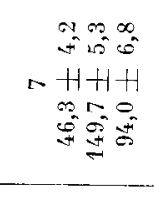 & 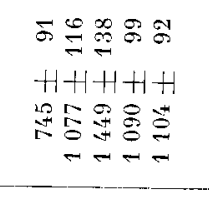 & $\begin{array}{l}20 \\
\infty \\
+1 \\
\infty \\
8 \\
0 \\
+1\end{array}$ & & 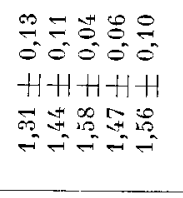 & $\begin{array}{l}0 \\
0 \\
0 \\
+1 \\
0 \\
0\end{array}$ \\
\hline & $\ddot{u}$ & 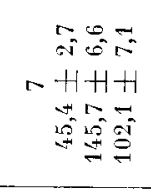 & 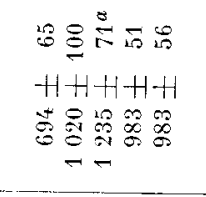 & $\begin{array}{l}20 \\
\dot{y} \\
H \\
0 \\
0 \\
\theta\end{array}$ & & 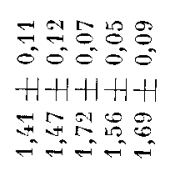 & 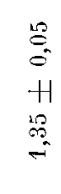 \\
\hline & D. & 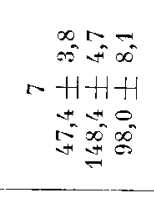 & 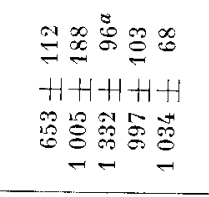 & 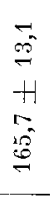 & & 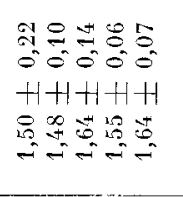 & $\begin{array}{l}\stackrel{0}{=} \\
= \\
+ \\
+ \\
\stackrel{5}{=}\end{array}$ \\
\hline & $\mapsto$ & 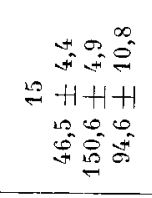 & 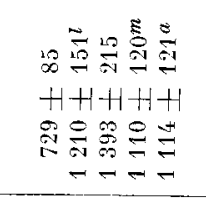 & $\begin{array}{l}8 \\
0 \\
+1 \\
\infty \\
0 \\
0\end{array}$ & & 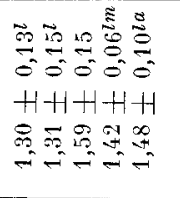 & $\begin{array}{l}\mathscr{8} \\
8 \\
+1 \\
\approx \\
\approx\end{array}$ \\
\hline & 营 & 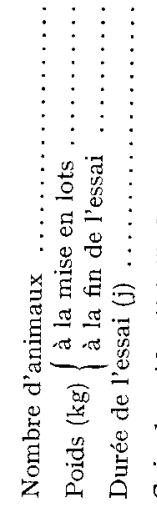 & 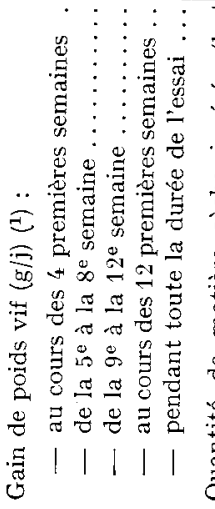 & 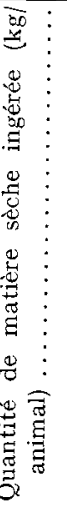 & 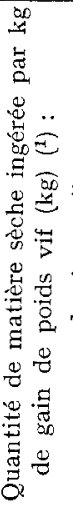 & 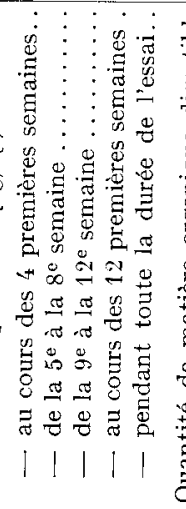 & 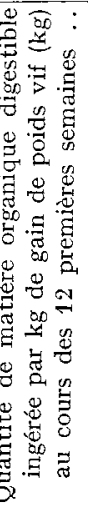 \\
\hline
\end{tabular}




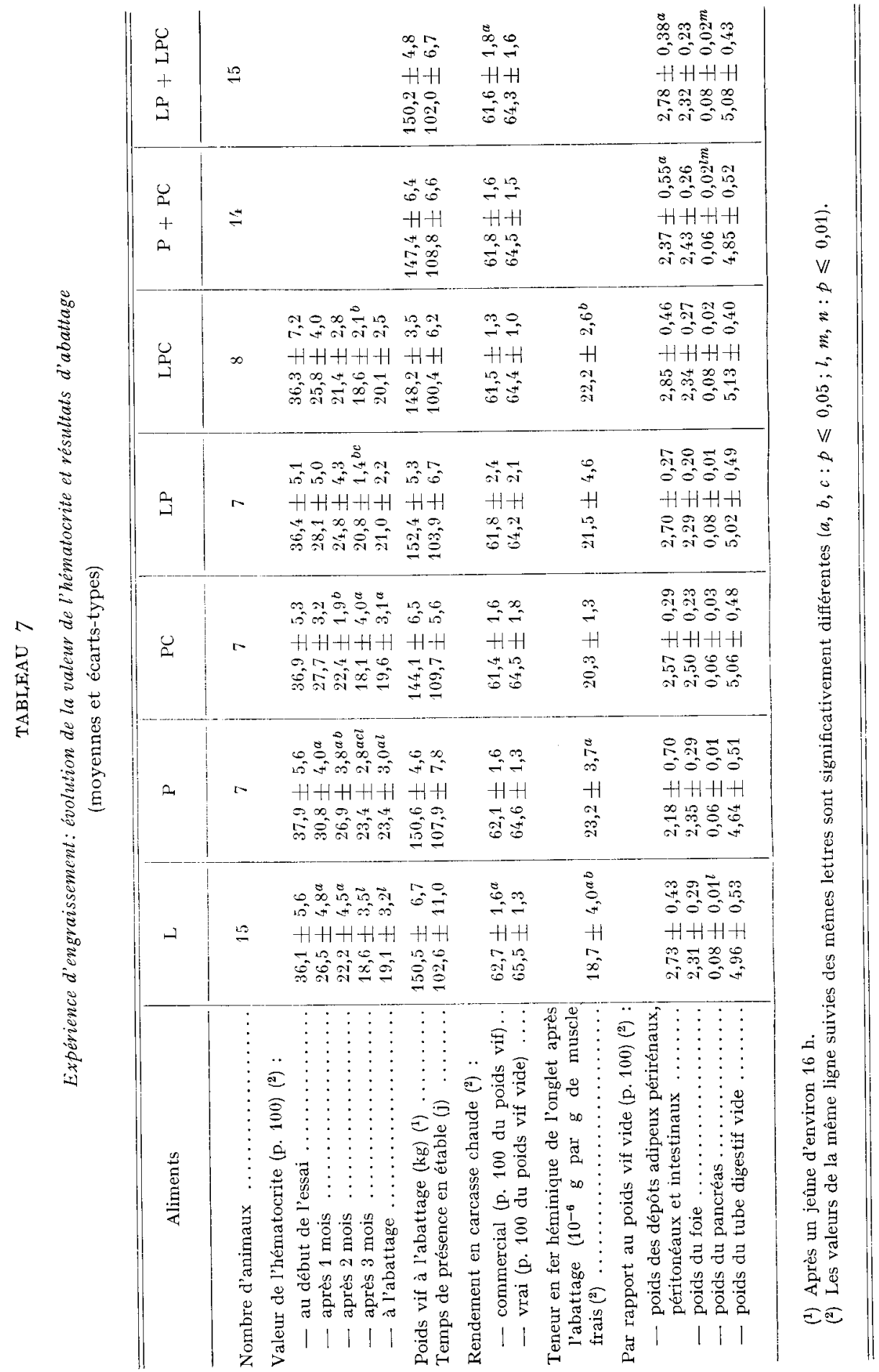


diminué fortement et ont toujours été semblables. L'état d'anémie des animaux était tel que des injections de fer dextrane $(500 \mathrm{mg}$ de fer) ont été effectuées chez certains d'entre eux dès la fin du $2^{e}$ mois ( $8 \mathrm{du}$ lot $\mathrm{L}_{1}, 3$ du lot PC et $4 \mathrm{du}$ lot $\left.\mathrm{L}_{\mathbf{P}} \mathrm{PC}\right)$. Ces injections expliquent peut être la légère élévation de la valeur de l'hématocrite observée avant l'abattage des animaux de ces 3 lots. La valeur de l'hématocrite des veaux recevant l'aliment $P$, contenant 24 p.p.m. de fer, a diminué plus faiblement et elle a été significativement supérieure $(p<0,05)$ à celles des veaux du lot $\mathrm{L}$ et du lot PC dès la fin du premier mois. L'évolution de la valeur de l'hématocrite des veaux recevant l'aliment I.P (I5 p.p.m. de fer) a été intermédiaire entre celles des veaux des lots $L$ et $P$, sans que les différences ne soient jamais significatives.

\section{Résultats d'abattage (tabl. 7).}

Le rendement en carcasse a été satisfaisant pour l'ensemble des lots. Le rendement vrai a été légèrement plus élevé chez les animaux du lot $\mathrm{L}$ que chez ceux des lots $\mathrm{P}+\mathrm{PC}(\mathrm{NS})$ et $\mathrm{LP}+\mathrm{LPC}(p<0,05)$. Chez les animaux du lot $\mathrm{P}+\mathrm{PC}$, le poids des dépôts adipeux périrénaux, péritonéaux et intestinaux, rapporté au poids vif vide, a été légèrement inférieur à celui obtenu chez les animaux des lots $\mathrm{L} P+\mathrm{LPC}$ $(p<0,05)$ et $\mathrm{L}$ (NS). L'état d'engraissement des carcasses a cependant été satisfaisant pour tous les animaux, à l'exception d'un animal du lot $\mathrm{L}$ et d'un autre du lot $P$, où il a paru insuffisant.

La coloration des carcasses a eu tendance à être un peu trop prononcée chez les animaux du lot $\mathrm{P}$ alors que, dans l'ensemble, elle a été très satisfaisante chez les animaux des autres lots. Ces résultats sont en accord avec ceux de la valeur de 1'hématocrite à l'abattage des animaux. Ils ne sont cependant que partiellement confirmés par le dosage du fer héminique dans les piliers latéraux du diaphragme. Cependant, le choix de ces muscles est lié à des considérations économiques, les muscles les plus représentatifs de la coloration de la carcasse étant, d'après CHARPENTIER (I964), les adducteurs et le couturier. Le poids du tube digestif et le poids du foie rapportés au poids vif vide, semblent avoir été plus élevés chez les veaux des lots PC et LPC que chez ceux des lots $P$ et LP (NS). Quant au poids du pancréas rapporté au poids vif vide, il a été plus faible $(p<0,0 \mathrm{I})$ chez les veaux du lot $\mathrm{P}+\mathrm{PC}$ que chez les veaux des lots L et LP + LPC.

\section{Test de dégustation.}

La viande provenant d'un animal du lot $\mathrm{L}$ a été jugée tendre ( $\mathrm{I} 7$ réponses), onctueuse (I6 réponses) et d'un goût agréable ( $I 7$ réponses). Celle provenant d'un veau du lot $\mathrm{P}$ a semblé moins tendre ( $\mathrm{I} 2$ réponses) et moins onctueuse (1o réponses). Elle a été jugée d'un goût agréable par I 3 personnes seulement, alors que 3 personnes lui ont reproché un goût insuffisant et une seule un goût trop prononcé. Ses défauts de tendreté et d'onctuosité ont probablement été responsables du fait qu'elle ait été moins appréciée, mais il ne semble pas que l'alimentation ait été à l'origine d'un goût particulier de cette viande. 


\section{DISCUSSION}

Le lait de remplacement utilisé dans 1'expérience de bilans était dépourvu de lait écrémé et, de ce fait, ne coagulait pas dans la caillette. Malgré l'accélération du transit digestif qui en résultait au niveau gastrique (GUILIOTEAU et al., travaux en cours), la fréquence des diarrhées est restée très faible. Ces résultats sont en accord avec ceux obtenus par Frantzen, Toullec et Mathieu (I97I) avec des laits de remplacement à base de lait écrémé rendus incoagulables, et par Toul_Ec et al., (I969) et PARUELLE et al., (I972) avec des laits de remplacement dont les protéines provenaient du lactosérum, du soja ou des levures d'alcanes. Il semble que la consistance des fèces soit davantage déterminée par l'origine et la technologie de préparation des protéines que par l'absence de coagulation du lait de remplacement (Toullec, Mathieu et Pion, I974).

Comme l'ont observé Frantzen, Toullec et Mathied (I97I), l'absence de coagulation de l'aliment a provoqué une baisse de la digestibilité apparente des matières azotées au cours du premier mois de vie des animaux. Ces résultats sont en accord avec ceux obtenus par Nol,er et al., (I956), Toullec et al., (I969), Paruelle et al., (I972) et Touliec, Mathieu et Pion (I974) avec des laits de emplacement dépourvus de lait écrémé. Le système digestif du très jeune Veau paraît donc insuffisamment adapté aux modifications entraînées dans la digestion par l'absence de coagulation des protéines. L'équipement enzymatique protéolytique, en particulier, est alors peu développé (Henschel, Hir et PORTER, I96I ; Ternouth, Siddons et Toothili, i97 ; Ternouth et Butrle, r973; Ternouth et Roy, I973). A partir de 1'âge d'un mois, la digestibilité apparente des protéines de poisson a été élevée ( 9 I p. IOo). Elle est légèrement supérieure à celle obtenue par VAN HELIEMOND (I967), pour une farine de hareng délipidée par 1'isopropanol ( 87 à 89 p. I00). Cela pourrait indiquer un effet favorable de l'hydrolyse enzymatique réalisée au cours de la préparation du concentré protéique ou bien une influence des espèces de poissons utilisées. La digestibilité apparente des protéines de poisson est cependant moins élevée que celle des protéines du lait écrémé séché par le procédé Spray (95 p. Ioo, d'après ToulifEC et MATHIEU, Ig6g) et celle des protéines du lactosérum 194 p. Ioo après concentration par ultrafiltration et séchage par le procédé Spray, d'après TouLLEC et al., résultats non publiés). Elle est en revanche supérieure à celle des protéines végétales, telles que celles des levures d'alcanes et surtout du soja (PARUElLE et al., I972), en raison des composés probablement peu digestibles (glucides complexes) qui les accompagnent.

Lors de l'expérience d'engraissement, le rendement de la moitié des protéines đu lait par un mélange dont 68 p. Ioo provenaient du poisson n'a pas notablement modifié le gain de poids vif des veaux. En revanche, le remplacement de la totalité des protéines du lait écrémé a eu un effet dépressif important sur la croissance, bien que celle-ci se soit maintenue à un niveau acceptable. En utilisant le même concentré protéique de poisson, SAINT-LAUREN'T et Brisson (I972) ont observé d'importantes diminutions dans la vitesse de croissance des veaux à l'engrais lorsque 80 p. roo des protéines alimentaires provenaient du poisson. Par ailleurs, chez le Veau d'élevage, Gorrilı, Nichorson et Power (I972) concluaient à la possibilité 
d'apporter $50 \mathrm{p}$. Ioo des protéines de l'aliment d'allaitement sous forme d'un concentré de protéines de poisson. Dans notre essai, l'efficacité alimentaire a été d'autant moins satisfaisante que le taux de substitution des protéines du lait écrémé était plus élevé.

Ces résultats peuvent être expliqués en partie par la plus faible digestibilité des protéines de poisson par rapport à celles du lait mais surtout par leur plus faible teneur en acides aminés indispensables et semi-indispensables et par le moins bon équilibre de ces derniers. Ainsi, l'aliment Poisson, utilisé dans l'expérience de bilans et l'aliment $P$, utilisé dans l'expérience d'engraissement, avaient des compositions très voisines. Compte tenu de leur supplémentation en méthionine, ils présentaient une légère carence en acides aminés aromatiques et/ou en tryptophane et en histidine, comme l'indiquaient les teneurs en acides aminés libres du sang des reaux qui recevaient ces 2 aliments (Patureau-Mirand, Prugnaud et Pion, i97 I ; Patureau-Mirand et al., I974). Ces résultats confirment ceux obtenus par GENsKOW, HaRshbarGer et WENDLANDT (I969), qui mettaient en évidence une carence en histidine et en tyrosine chez des veaux recevant des aliments d'allaitement contenant de la farine de poisson. Il est possible que ces carences aient été accentuées par l'élimination de la fraction protéique demeurée insoluble lors de la préparation du concentré protéique ; ainsi, chez le porcelet, SÈve et AUMAITRE (r973) ont obtenu une croissance plus rapide, malgré une digestibilité moins élevée des protéines, lorsque cette fraction n'était pas éliminée. Le coefficient de rétention apparent de l'azote de l'aliment Poisson a néanmoins été élevé. La composition en acides aminés disponibles de cet aliment était donc relativement satisfaisante, malgré le léger déséquilibre signalé. Il a cepenđant conduit à des quantités d'azote apparemment retenues par $\mathrm{kg}$ de gain de poids vif anormalement élevées. L'expérience de bilans s'est déroulée dans une étable non chauffée, au cours d'un hiver relativement rigoureux et les animaux avaient développé un pelage important. Il se peut, en outre, qu'il y ait eu des pertes d'urine.

Par ailleurs, nos observations paraissent confirmer l'absence de goût particulier de la viande des veaux ayant ingéré des protéines de poisson, déjà constatée par SAINT-LAUREN'T et BRISSON (I972). Par rapport à d'autres sources de protéines de remplacement (PARUELLE et al., I972), le concentré protéique de poisson utilisé avait une teneur en fer relativement faible ( 80 p.p.m. environ), et les aliments les plus riches en protéines de poisson (aliments $P$ et $P C$ ) ne contenaient que 25 p.p.m. de fer. De ce fait, la coloration des carcasses provenant des veaux ayant reçu l'aliment $\mathrm{P}$ n'a été que légèrement plus foncée. Le dérivé de 1'E'D'TA (Nourycal 25) contenu dans les aliments PC et LPC semble avoir limité l'utilisation du fer alimentaire et pourrait permettre d'obtenir des carcasses peu colorées. Les veaux ayant reçu ces aliments présentaient un état d'anémie comparable à celtui des veaux ayant reçu l'aliment $\mathrm{L}$ à base de protéines de lait. Malgré cela, la vitesse de croissance et l'efficacité alimentaire n'ont pas été fortement affectées par la supplémentation en complexant, alors que le poids du foie des veaux l'ayant reçu a paru plus élevé. Des essais complémentaires devraient être entrepris en vue d'envisager l'utilisation d'un tel produit.

En conclusion, les protéines issues du poisson blanc et ayant subi une hydrolyse enzymatique ménagée semblent intéressantes à incorporer dans les aliments d'allaitement destinés au veau préruminant à l'engrais. Leur digestibilité est élevée et leur 
équilibre en acides aminés indispensables est relativement satisfaisant. Associées au lactosérum, elles pourraient apporter environ la moitié des protéines alimentaires sans provoquer de baisse importante dans les performances des animaux. Dans ces conditions, leur teneur en fer parait suffisamment faible pour permettre d'obtenir une viande peu colorée lors de l'abattage. L'emploi de composés à base de dérivés de 1'EDTA permettant de complexer le fer alimentaire pourrait être envisagé sous réserve d'essais complémentaires. Il serait également judicieux d'étudier 1'utilisation par le Veau de concentrés protéiques de poisson préparés selon d'autres procédés technologiques.

Reçu pour publication en juin 19rt.

\section{SUMMARY}

\section{UTILIZATION OF PROTEIN BY PRERUMINANT FATTENING CALVES. II. - UTILIZATION OF FISH PROTEIN}

AND EFFECT OF ADDING AN IRON CHELATING AGENT

The utilization of fish protein in milk replacers for fattening preruminant calves was stuclied. The protein was supplied in form of concentrate, obtained by mild enzymatic hydrolysis of white fish, and containing about 90 p. Ioo crude protein and 80 p.p.m. iron.

During the first experiment (table 5), 3 calves, kept in balance cages, received a milk replacer in which $73 \mathrm{p}$. Ioo of the proteins were supplied from fish, and the rest in form of whey and a methionine supplement. The frequency of days with diarrhoea was very low ( 2 p. Ioo) that of days with loose faeces was very high $(74 \mathrm{p}$. I0o). The apparent digestibility of the dietary crude protein increased during the first month of age, from $82 \mathrm{p}$. Ioo between I 5 and $2 \mathrm{I}$ days to $89 \mathrm{p}$. I 00 between 24 and 34 days. Thereafter, it remained high and constant ( 92 p. Ioo) between 37 and 105 days. This increase might account for an insufficient adaptation of the digestive system in the very young calf to the change in the gastrointestinal transit due to the absence of protein coagulation in the abomasum. The apparent retention coefficient of nitrogen was high, showing that the balance of available essential amino acids in the dietary proteins was satisfactory.

During the second experiment (tables 6 and 7$), 48$ calves were kept in individual pens from the age of about one week and until a slaughter liveweight of about I50 $\mathrm{kg}$. After one week of adaptation, they were distributed into 5 groups each receiving one of the 5 milk replacers considered. In the first milk replacer ( 16 calves), the proteins proceeded exclusively from milk. In the next two ( 2 groups of 8 calves), they came for 68 p. Ioo from fish and the rest from whey and a methionine supplement. An EDTA component was added to one of these replacers so as to chelate (complex) the iron in excess from the fish. The composition of the two other diets (2 groups of 8 calves), was intermediate between the first milk replacer and each of the two following ones. All the diets were well eaten and the health of the animals was satisfactory.

The diet containing milk protein, and those with an intermediate composition gave similar liveweight gains (about I $100 \mathrm{~g} / \mathrm{day}$ ) significantly higher than those obtained with the diets in which $68 \mathrm{p}$. I oo of the proteins proceeded from fish ( $1009 \mathrm{~g} / \mathrm{day}$ ). The dry matter intake per $\mathrm{kg}$ liveweight gain significantly increased with the substitution level of the milk proteins (I.48, $\mathbf{I} .55$ and $\mathbf{I} .67 \mathrm{~kg}$, respectively). The dressing out percentage and fatness of the carcasses were satisfactory in all cases. The meat colour was a little too dark in the calves receiving rich fish protein diets without addition of an iron chelating agent, and satisfactory in all the other cases. According to the hematocrit value of the calves, the iron chelating agent seems to have limited the utilization of dietary iron.

The proteins from white fish having been subjected to a mild enzymatic hydrolysis seem to be well fitted for incorporation into milk replacers. Associated with whey, they might supply about half of the dietary proteins without lowering the performances of the calves. 


\section{RÉFÉRENCES BIBLIOGRAPHIQUES}

Blaxter K. L., I962. The energy metabolism of ruminants. Hutchinson, London, 332 p.

Blaxter K. L., Wood W. A., 1953. Some observations on the biochemical and physiological events associated with diarrhoea in calves. Vet. Rec., 50, 889-892.

Charpentier J., ig64. Comparaison de quelques méthodes d'appréciation de la couleur du veau de boucherie. Ann. Zootech., 13, I03-I 4.

Frantzex J. F., Tovllec R., Mathieu C.-M., I97i. Influence de la coagulation sur l'utilisation digestive des matières azotées du lait. $X^{\mathrm{e}}$ Congrès International de Zootechnie, 17-23 juillet, Versailles.

Genskow R. I), Harshbarger K. E., Wendlandt R. M., r968. Observations on vitamin deficiencies in calves fed a milk replacer containing low-ash fish meal (abstr.). J. Dairy Sci., 51, 973.

Genskow R. 1), Hakshibarger K. E., Wendlandt R. M., ig69. Iiffect of feeding fish protein concentrate in milk replacers on plasma free amino acid values (abstr.). J. Dairy Sci., 52, 993.

Gorrill A. I). L., Nicholson J. W. G., Power H. F., I972. Effects of milk, fish, and soybean protein in milk replacers, and feeding frequency on performance of dairy calves. Can. J. Anim. Sci. 52, $32 I-328$.

Harshbarger K. E., Gelwicks T. J., I965. Fish flour as a protein source in inilk replacers for dairy calves (abstr.). J. Dairy Sci., 48, 788 .

Henschel M. J., Hill W. B., Porter J. W. G., I96I. The development of proteolytic enzymes in the abomasum of the young calf. Proc. Nutr. Soc., 20, XI.

Hornser H., r956. The colour of cooked pork. I. Estimation of the nitric oxide-haem pigments. $J$. Sci. Food A gric., 7, 534-540.

Hubir J. T., Slade L. M., 1967. Fish flour as a protein source in calf milk replacers. J. Dairy Sci., 50, $1296-1300$.

KhammotMa M., Arbogast $F$, I97o. La troisième génération des aliments d'allaitement : le "lait sans lait". Rev. Med. Vét., 121, 59.3-609.

Makdani D. D., Huber J. T., Michel R. L., I97I. Nutritional value of i,2-dichloroethane extracted fish protein concentrate for young calves fed milk replacer dicts. J. Dairy Sci., 54, 886-892.

Michi: R. L., Makdani D. D., Huber J. T., Sculthorpli A. E., I972. Nutritional myopathy due to vitamin $\mathrm{E}$ deficiency in calves fed fish protein concentrate as the sole source of protein. $J$. Dairy Sci., 55, 498-506.

Noller C. J., Ward C. M., Machilliati A. D., Huffman C. F., Duncan C. W., i958. The effect of age of the calf on the availability of nutrients in vegetable milk-replacer ration. J. Dairy Sci., 39, $1288-\mathrm{I} 298$.

Parulle J.-L., Toullec R., Frantzen J.-F., Mathieu C.-M., r972. Utilisation des protéines par le Veau préruminant à l'engrais. I. Utilisation digestive des protéines de soja et des levures d'alcanes incorporées dans les aliments d'allaitement. Ann. Zootech., 21, 319-33I.

Patureal-Mirand P., Prugnaud J., Pion R., I97I. Influence de la nature des protéines des aliments d'allaitement sur l'amino-acidémic libre du Veau préruminant. $X$ e Congrès Intern. Zootech. I7-23 juillet. Versailles.

Patureat-Miraxd P., Toullec R., Paruelle J.-L., Prugnaud J., Piox R., rg74. Influence de la nature des matières azotées des aliments d'allaitement sur l'amino-acidémie du Veau préruminant. I. Matières azotées du lait, du lactosérum, du poisson et des levures d'alcanes. Ann. Zootech., 23, $343-358$.

Pion R., Falcosveau G., I966. Les acides aminés des protéines alimentaires. Méthodes de dosage et résultats obtenus. Amino-acides, Peptides, Protéines, Cahier no 6, 158-175. A. E. C., Commentry. Pion R., I971, Composition en acides aminés des aliments. Ind. Alim. Anin., 6, 29-36.

Ravix A. M., 1972. Nutritional effects of including different levels and sources of protein in milk for calves. J. Sci. Fd. Agric., 23, 517-526.

Seve B., Avmattre A., I973. Utilization of soluble fish protein concentrates in dry diets for young piglets weaned at 12 days of age. European Nutr. Conf., 33, Cambridge.

Saint-Latrext G. J., Brisson (i. J., 1972. Nutritive value of a lipC for young calves. Can. J. Anim. Sci., 52, 585 (Abstr.).

Siump P., Schretder H. A. W., I969. Jetermination of tryptophan in foods. Analyt. Biochem., 24, I $82-186$.

Ternocth J. H., Buttle H. L., i973. Concurrent studies on the flow of digesta in the duodenum and of exocrine pancreatic secretion of calves. The collection of the exocrine pancreatic secretion from a duodenal cammula. Hr. J. Nutr., 29, $3^{87-397 .}$

Ternotth J. H., Roy J. H. 13., 1973. The effect of diet and feeding technique on digestive function in the calf. Ann. Rech. vétér., 4, I9-30.

Ternotth J. H., Srddons R.C., Toothill J., I97I. Pancreatic secretion in the milk-fed calf. Proc. Nutr. Soc., 30, 89 A. 
Toullec R., Mathieu C.-M., r969. Utilisation digestive des matières grasses et de leurs principaux acides gras par le Veau préruminant à l'engrais. Influence sur la composition corporelle. Ann. Biol. anim. Bioch. Biophys., 9, I39-工6o.

Toullec R., Mathieu C.-M., Pion R., 1973. Utilisation des protéines du lactosérum par le Veau préruminant à l'engrais. II. Digestibilité et utilisation pour la croissance. Ann. Zootech. 23 75-87.

Toullec R., Mathieu C.-M., Vassal L., Pron R., ig69. Utilisation digestive des protéines du lactosérum par le Veau préruminant à l'engrais. Ann. Biol. anim. Bioch. Biophys., 9, 661-664.

Toullec R., Patureau-Mirand P., Paruelie J.-L., Guilhermet R., I973. Utilisation des protéines par le Veau préruminant à l'engrais. Bull. Soc. Sci. Hyg. Alim., 61, 57-92.

VAa Hellemond K. K., I967, cité par Norrman E., I97r. Fish protein concentrate in milk replacers. Proc. Int. Milk Replacer Symp. 26-27 mai, Zurich, I4I-I48.

Wendlandt R. M., Harshbarger K. E., Genskow R. D., Ig68. Growth response of calves fed milk replacers containing fish meal. J. Dairy Sci., 51, 972-973. (Abstr.). 